\title{
Numerical Modeling of Antennas with Mechanically/MEMS-Enabled Moving Parts
}

\author{
Kohei Yamagata $^{(1)}$, Michiko Kuroda $^{(1)}$, Manos M. Tentzeris ${ }^{(2)}$ \\ ${ }^{(1)}$ Tokyo University of Technology,Hachioji, Tokyo, 198-0982, Japan \\ ${ }^{(2)}$ School of ECE, Georgia Institute of technology, Atlanta, GA 30332-250, \\ U.S.A. \\ kuroda@cc.teu.ac.jp
}

\begin{abstract}
In this paper, a numerical technique for the design, optimization and realization of antennas with mechanically/MEMS-enabled moving parts is proposed. The accuracy of this technique is evaluated through the calculation of the numerical results that express the relation between the velocity of the moving part and the S11 parameters.
\end{abstract}

Keywords: RF MEMS, moving boundary problem, body fitted grid generation method with moving boundaries, FD-TD method

\section{Introduction}

RF MEMS technology has rapidly developed, due to its inherent low-power and miniaturization advantages over $p$-i-n diode or FET switches[1]-[3]. An accurate knowledge of the electromagnetic field evolution around a moving or rotating body is required for the realization of new optical and microwave devices, such as the RF-MEMS structures used in phase-shifters, couplers or filters, as well as reconfigurable antennas. Computational technique for the moving boundary problems have been pursued mainly in heat and fluid flow area. In this paper, we propose a numerical approach for the analysis of this type of problems that alleviates the limitations of the conventional time-domain techniques [4]. By using a transformation with a time factor, it is possible to apply the grid generation technique of [5] to the time-domain analysis of the moving object. This is a feature that makes the new method especially useful for analyzing moving objects of arbitrary shape. In this paper, this simulation method is applied to the analysis of a patch antenna with moving parts, that aims to extend the design of [6] to reconfigurable center frequencies. The numerical results that express the relation between the velocity and the S11 parameters are shown to validate the proposed approach. 


\section{Two Dimensional Micromachined Patch Antenna Model}

The geometry to be considered here is shown in Fig.1. Without loss of generality and as a proof of concept, under the combined effect of the mechanical and the electrical force, the top patch/membrane is assumed to move with constant velocity for a specific time vertically and/or horizontally to modify the central frequency and the bandwidth. This effect is equivalent to the motion of the with constant velocities to the opposite direction. It is easier to simulate the motion of the bottom plate, because it's a uniform metal (single boundary condition). On the opposite, the simulation of the top membrane's motion would be more tedious since it would involve the combination of two boundary conditions (dielectric/metal). For the two-dimensional TMpropagation case, there will be only $\mathrm{Ex}, \mathrm{Ey}, \mathrm{Hz}$ nonzero components with a time variation given by the following equations

$$
\begin{aligned}
& \frac{\partial H_{z}}{\partial t}=\frac{1}{\mu}\left(\frac{\partial E_{x}}{\partial y}-\frac{\partial E_{y}}{\partial x}\right) \\
& \frac{\partial E_{x}}{\partial t}=\frac{1}{\varepsilon}\left(\frac{\partial H_{z}}{\partial y}-J_{x}\right) \\
& \frac{\partial E_{y}}{\partial t}=-\frac{1}{\varepsilon}\left(\frac{\partial H_{z}}{\partial x}+J_{y}\right)
\end{aligned}
$$

where $\varepsilon, \mu$ are the constitutive parameters of the respective media. In Fig.1, the configuration of the physical region is shown. The bottom plate is assumed to move to the $x, y$ directions with velocities $v$ and $u$, respectively. Using a coordinates' transformation technique, the time-changing physical region $(x, y, t)$ can evolve to a time-invariant computational domain $(\xi, \eta, \tau)$. $\mathrm{n}, \mathrm{m}$ are the indices of each subregion in $x$ - and $y$-direction. The number of subregions depends on the geometry of the moving parts of the geometry. The transform equations between the physical and the computational regions are chosen as :

$$
\begin{aligned}
& \xi=\alpha_{n-1} \frac{x-h_{n-1}(t)}{h_{n}(t)-h_{n-1}(t)}+\xi_{n-1} \\
& \eta=\beta_{m-1} \frac{y-g_{m-1}(t)}{g_{m}(t)-g_{m-1}(t)}+\eta_{m-1} \\
& \tau=t \\
& h_{n}(t)=x_{n}+v t \\
& g_{m}(t)=y_{m}+u t
\end{aligned}
$$

where $\mathrm{n}=1,2,3,4,5 \mathrm{~m}=1,2,3,4,5$ and $h_{0}(t), \quad h_{1}(t), \quad h_{2}(t), \quad h_{3}(t), \quad h_{4}(t), \quad h_{5}(t)$, $g_{0}(t), \quad g_{1}(t), \quad g_{2}(t), \quad g_{3}(t), \quad g_{4}(t), g_{5}(t)$ are written assuming that the plate velocities remain constant for the whole time of their motion. $\alpha_{n-1}, \beta_{m-1}$ are coefficients to normalize the computational region. The coordinates $x_{1}, x_{2}, x_{3}, x_{4}$, and $y_{1}, y_{2}, y_{3}, y_{4}$ represent the initial positions of the plates. The 
functions $h_{1}(t), h_{2}(t), h_{3}(t), h_{4}(t), g_{1}(t), g_{2}(t), g_{3}(t), g_{4}(t)$ describe the movement along the $x$ and $y$ axis, respectively, and allow for the realization of a rectangular grid with stationary boundary conditions, where $h_{0}(t)=0, h_{5}(t)=L_{x}$, $g_{0}(t)=0, g_{5}(t)=L_{y}$. The partial time-derivatives in the transformed domain $(\xi, \eta, \tau)$ can be expressed in terms of the partial derivatives of the original domain $(x, y, t)$ using eqs.(4)-(8). The FDTD technique can provide the timedomain solution of the rectangular $(\xi, \eta, \tau)$ grid. The stability criterion in this case is chosen as $c \Delta t \leq \delta / \sqrt{2}$, where, assuming the grid is uniformly discretized in both directions. In general, $\delta$ is a the minimum space increment (minimum cell size) for $x$ and $y$ directions.

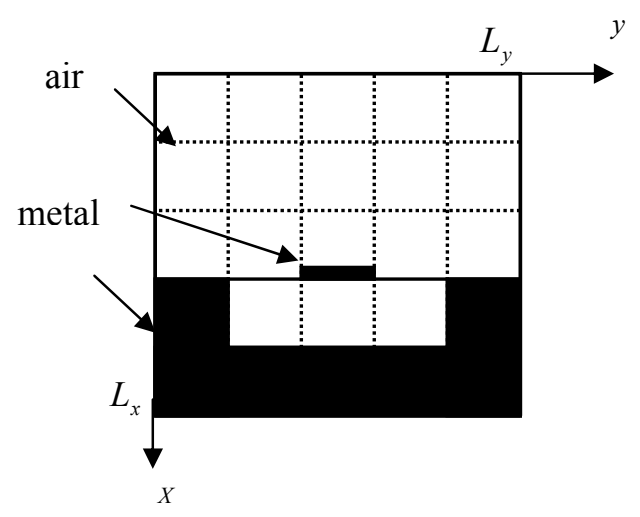

Fig. 1. Side View of the Model

\section{Numerical Results}

To validate this approach, numerical results are calculated for a microstrip antenna with the bottom plate moving only to the $x$-direction. The grid includes $200 \times 200$ cells and, input frequency is $f=10 \mathrm{GHz}$ and $L_{x}=L_{y}=L_{z}=L=3 \lambda$, $\Delta x=\Delta y=L / 200, \Delta t=L / 800 c$, where $c$ is a velocity of light. The initial plate separation is $L / 5$ and the grid is terminated with Mur's absorbing boundary conditions. Fig 2 shows the time dependence of the transient capacitance for the velocity values in the range of $v=-5 \times 10^{-3} c$ to $v=-7 \times 10^{-3} c$. As a reference reason, the stationary value is shown. The stationary value has a good agreement with the theoretical result. It is easy to perform an accurate analysis of the transient response of the structure and predict the ringing parasitic effects. It is clear that the transient effect is more pronounced for the higher values of velocity. Table I shows the variation of the center frequency for the different velocity values for the antenna with moving plates for a motion lasting 1000 time-steps. It is observed that the difference velocity value makes a different resonant frequency. The stationary antenna has a resonant frequency around 10 $\mathrm{GHz}$. There can be seen a shift of the resonant frequency for the moving case. 


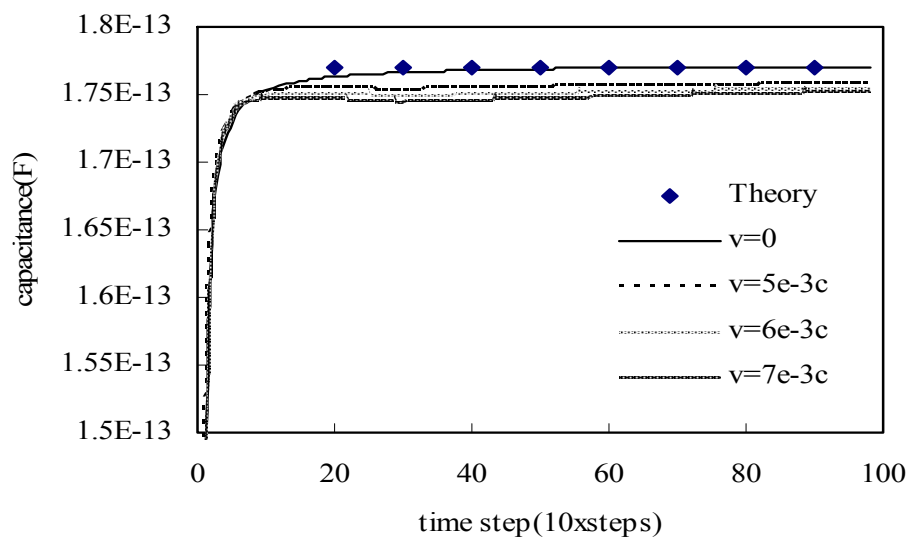

Fig2 Transient capacitance for each velocity values

Table I The variation of the center frequency for the different velocity values

\begin{tabular}{|l|l|}
\hline velocity & Resonant Frequency \\
\hline $1.1 \times 10^{-3} c$ (approaching) & $7.63 \mathrm{GHz}$ \\
\hline 0 & $8.02 \mathrm{GHz}$ \\
\hline $1.1 \times 10^{-3} c$ (departing) & $8.78 \mathrm{GHz}$ \\
\hline
\end{tabular}

\section{Conclusions}

In this paper, a time-domain numerical technique for the analysis and design of a reconfigurable patch antenna with mechanically/MEMS enabled moving parts is presented. For the validation of this technique, the numerical results of the relation between the capacitance and the velocity of the motion are shown. And also the relation between the central (resonant) frequency and the velocity of the plate and the direction of the motion is derived by using the proposed technique. A shift of the resonant frequency can be observed for different velocities. By using this technique, it is possible to analyze more complicated reconfigurable structures with moving boundaries in one, two or three directions.

\section{References}

[1] Gabriel M. Rebeiz, "RF MEMS Theory, Design and Technology", John Wiley \& Sons, New Jersey, 2003.

[2] Alekander Dec, et-al, "Micromachined Electro-Mechanically Tunable Capacitors and Their Applications to Re IC's “, IEEE Trans. MTT, Vol.46, No.12, pp2587-2596, Dec. , 1998

[3] Alekander Dec, et-al, "Microwave MEMS-Based Voltage=Controlled Oscillators", IEEE Trans MTT, Vol.48, No.11,pp1943-1949, Nov.,2000.

[4] Michiko Kuroda, Noriyuki Miura, Manos M. Tentzeris, “ A Novel Numerical Approach for the Analysis of 2D MEMS-Based Variable Capacitors Including the Effect of Arbitrary Motions", ACES Journal, Vol. 19, No.1b, pp133-138, March 2004.

[5]J. F. Thomson, “ Numerical Grid Generation ”, North Holland, Amsterdam, 1985

[6] E. Y. Tsai, A. Mbacon, M. Tentzeris, J. Papapolymerou, "Design and Development of Novel Micromachined Patch Antenna for wireless applications", Proc. of 2002 APMC, ppII821-824, Nov. 2002, Kyoto, JAPAN 